\title{
FACTEURS PREDICTIFS DE L'EFFICACITE ABLATIVE DE L'IODE 131 DANS LES CANCERS DIFFERENCIES DE LA THYROÏDE
}

\author{
M. GUEZGUEZ, K. CHATTI, M. NOUIRA, S. ZROUR **, M. BEN FRADJ, R. SFAR *, \\ A. BOUARS, K. BEN ALI *, S. AJMI, H. ESSABBAH \\ SERVICE DE MÉDECINE NUCLÉAIRE CHU SAHLOUL SOUSSE. \\ *UNITÉ DE RECHERCHE TIM, FACULTÉ DE MÉDECINE MONASTIR. \\ ** SERVICE DE RHUMATOLOGIE CHU F.BOURGUIBA MONASTIR.
}

\begin{abstract}
La prise en charge des cancers différenciés de la thyroïde (CDT) comporte souvent une radiothérapie métabolique à l'iode131 (IRA-thérapie). Le but de ce travail est d'évaluer le caractère prédictif des différents éléments anatomopathologiques, de la classification pTNM et de la stadification pronostique sur l'activité ablative requise d'iode131.

Notre travail est une étude analytique rétrospective portant sur 275 cas de CDT ayant subit une thyroïdectomie totale. Tous ces patients ont eu une ou plusieurs activités ablatives. Nous avons cherché - au moyen d'une analyse statistique par test de Khi2 ou test Anova - toute corrélation entre les éléments de l'examen anatomopathologique de la tumeur, la classe pTNM, le stade pronostique correspondant d'une part et l'efficacité de l'irathérapie ablative d'autre part.

Dans notre série, une activité ablative plus élevée est nécessaire lorsque la taille du foyer tumoral dépasse les $6 \mathrm{~cm}$ $(p=0,012)$, en cas de dépassement de la graisse péri thyroïdienne $(p<10-3)$ ou d'envahissement ganglionnaire multi-compartimentale $(p<10-3)$.

Un constat comparable est trouvé avec la classification pTNM. L'activité ablative augmente lors du dépassement du stade T3 $(p=0,002)$ et $N 1 a(p=0,039)$. L'activité ablative requise n'est cependant pas corrélée à la stadification pronostique TNM.

En conclusion, certains éléments anatomopathologiques sont prédictifs de l'efficacité de l'IRAthérapie ablative. Une taille tumorale inférieure à $6 \mathrm{~cm}$, l'atteinte lymphatique non pluri-compartimentale et l'absence d'invasion de la graisse péri-thyroïdienne prédisent une meilleure efficacité ablative de l'iode131. Cette efficacité peut être traduite dans la classification pTNM, ce qui correspond à $\mathrm{T}<\mathrm{T} 3$ et $\mathrm{N}<\mathrm{N} 1 \mathrm{~b}$, mais pas dans le stade pronostique de la tumeur.

Mots-clés : Cancer de la thyroïde ; lode 131 ; Efficacité ablative ; Caractéristiques anatomopathologiques.
\end{abstract}

lodine131 is often used in the management of thyroid's differentiated carcinoma (TDC). The aim of this paper is to establish the predictive value of anatomopathologic parameters, pTNM classification and prognostic staging on required ablative activity.

We studied retrospectively cases of 275 patients with TDC. All patients was treated with radioactive iodine after total thyroidectomy. Data was analyzed using statistical tools (Khi2 or ANOVA tests).

Our study found that highest activities are required when tumor size exceeds $6 \mathrm{~cm}(p=0.012)$, when peri-thyroid fat was invaded $(p<010-3)$ or when many cervical lymph node compartments are invaded $(p<010-3)$.

A comparable report is found with pTNM classification; in fact ablative activity grows beyond T3 $(p=0.002)$ and N1a $(p=0.039)$. No correlation was found with TNM prognostic staging.

In conclusion, our study shows that we can predict a better ablative efficiency of iodine131 when tumor size is less than $6 \mathrm{~cm}$ and when lymphatic invasion involved in most one compartment and when the integrity of peri-thyroid fat is respected which corresponds in pTNM classification to $\mathrm{T}<\mathrm{T} 3$ and $\mathrm{N}<\mathrm{N} 1 \mathrm{~b}$ classes.

Keywords : Thyroid cancer ; lodine 131 ; Ablative efficiency ; Anatomopathologic characteristics.

\section{INTRODUCTION}

Les cancers différenciés de la thyroïde (CDT) sont des tumeurs malignes qui se développent aux dépens du tissu thyroïdien. Les CDT sont rares représentant moins de $1 \%$ de tous les cancers (1). Chez ces patients, le traitement principal est chirurgical et consiste en une thyroïdectomie totale. Ce traitement peut être complété par une radiothérapie métabolique à l'iode 131. L'un des objectifs visés est de détruire tout reliquat thyroïdien résiduel après l'acte chirurgical. Les données disponibles actuellement sur l'utilisation post opératoire de l'iode 131 sont controversées entre l'indication systématique $(2,3,4,5)$ et l'indication selon les bénéfices potentiels attendus d'un tel traitement. De tels bénéfices sont rattachés aux risques en terme de morbidité et de mortalité $(6,7,8,9,10,11)$.

L'activité d'iode 131 requise pour la destruction du reliquat est variable et semble dépendre de plusieurs facteurs. L'acte chirurgical et le résultat de l'examen anatomopatho- 
logique semblent des éléments clefs de cette efficacité. Le rôle de la classification clinique (TNM) ou au mieux anatomopathologique (pTNM) et de la stadification qui en découle est bien établi dans l'évaluation du pronostic (12). Nous nous sommes proposés, par cette étude, d'évaluer le caractère prédictif des différents éléments anatomopathologiques, de la classification TNM et de la stadification pronostique sur l'activité ablative requise d'iode 131.

\section{MATÉRIEL ET MÉTHODES}

\section{- Type d'étude}

Notre travail est une étude rétrospective portant sur 275 cas de carcinome différencié de la thyroïde ayant subit une thyroïdectomie totale, puis suivis dans notre service entre 1992 et 2004. Notre population est composée de 41 hommes et 234 femmes. L'âge moyen est de 42,3 ans (extrêmes 7 à 85 ans). Tous ces patients ont eu une ou plusieurs activités ablatives.

\section{- Paramètres de l'étude}

Les données sont recueillies à partir des dossiers du service de médecine nucléaire et des comptes rendus de l'acte opératoire et de l'examen anatomopathologique. Nous avons inclus dans notre étude tous les patients présentant un CDT (papillaire ou vésiculaire), documenté par un compte rendu anatomopathologique complet et chez qui un reliquat thyroïdien aurait été identifié sur le Balayage corps entier (BCE) initial à l'iode 131.

De cette étude nous avons exclu les patients perdus de vue après le premier BCE.

Les principales variables étudiés portent sur l'acte chirurgical (le type et l'étendue du geste chirurgical), les résultats de l'examen anatomopathologique (siège, nature, taille de la tumeur, multifocalité du cancer et extension tumorale), la classification de la maladie (TNM, pTNM et la stadification pronostique) ainsi que les paramètres du traitement par l'iode 131 (activités cumulées jusqu'à disparition du reliquat et délais de disparition du reliquat).

\section{- Traitement des données}

L'analyse statistique des données recueillies est réalisée au moyen de logiciel d'analyse statistique. Cette analyse a pour objectif d'identifier toute corrélation entre les données de l'examen anatomopathologique cités plus haut et les paramètres d'efficacité ablative de l'iode 131. L'étude des corrélations est faite au moyen de test de Chi 2 et de test ANOVA. La différence est considérée comme significative pour toute valeur de $p$ inférieure à 0,05 .

\section{RESULTATS}

\section{- Résultats de l'examen anatomopathologique}

Dans $83,3 \%$ des cas (229 patients), la tumeur était de nature papillaire. Les tumeurs vésiculaires ne représentaient que $16,7 \%$ des cas (46 patients). La taille tumorale variait du microcarcinome qui est retrouvé dans 39 cas
(14,2\%), à une taille dépassant les $5 \mathrm{~cm}$ chez 36 patients (13\%). Le nombre de foyers néoplasiques variait également d'un foyer tumoral unique dans $59,3 \%$ des cas (163 patients) à plus de 10 foyers. La multifocalité représente $40,7 \%$ des cas.

L'absence d'envahissement local est constatée dans $61,5 \%$ des cas (169 patients). Sa présence ne représente que $38,5 \%$ des cas (106 patients). II s'agit d'une simple effraction capsulaire dans $20,4 \%$ (56 patients) et d'une extension à la graisse péri-thyroïdienne et même au delà dans $18,1 \%$ (50 patients). Un envahissement ganglionnaire est retrouvé chez 122 patients (44,4\%). II s'agit d'une atteinte unicompartimentale dans $28,4 \%$ des cas et pluricompartimentale dans $16 \%$ des cas. Les cas initialement métastatiques ne représentent que $5,5 \%$ des cas.

\section{- Influence des éléments anatomopathologiques sur l'activité ablative :}

Type histologique : L'activité ablative moyenne est de $5,68 \mathrm{GBq}$ en cas de carcinome papillaire. Dans la forme vésiculaire, cette activité moyenne devient 5,32GBq. La différence entre les deux moyennes est non significative. Le délai de disparition du reliquat diffère peu entre les deux groupes : 16 mois en moyenne pour le type papillaire et 17 mois pour le type vésiculaire. Cette différence n'est pas statistiquement significative.

Taille tumorale : Pour une taille tumorale inférieure à $5 \mathrm{~cm}$, l'activité ablative requise est le plus souvent inférieure à $3,7 \mathrm{GBq}$ (64\% des cas). Cette tendance s'inverse pour des tailles tumorales au delà de $6 \mathrm{~cm}$ avec des activités ablatives plutôt supérieures à $3,7 \mathrm{GBq}$ ( $58 \%$ des cas). La différence entre les deux groupes est statistiquement significative $(p=0,012)$ (figure1).

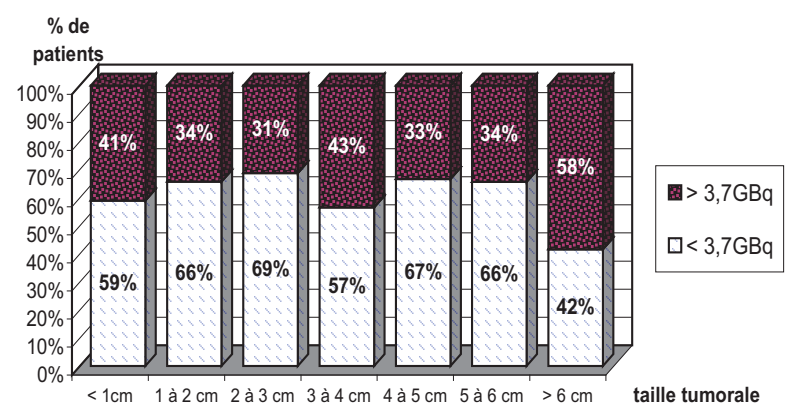

Fig. 1 : Pourcentage de cas d'ablation de reliquat selon que l'activité administrée est inférieure ou supérieure à 3,7GBq en fonction de la taille tumorale

- Nombre de foyers tumoraux : L'activité ablative nécessaire est plus importante dans les formes multifocales que dans les formes unifocales (figure 2). Cette différence n'est cependant pas significative. Le délai de disparition du reliquat est peu différent entre les formes multifocales (16 mois) et unifocales (16,3 mois). 


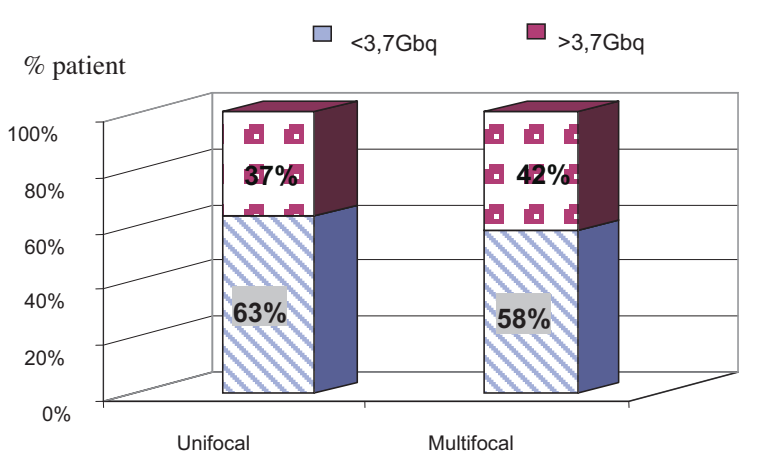

Fig. 2 : Activité ablative et nombre de localisations tumorales (uni ou multifocale)

- Envahissement locorégional : Les patients inclus dans cette étude ont été réparties en 4 groupes : Groupe 1 comprend les patients sans invasion locorégional, le groupe 2 ceux présentant une simple effraction capsulaire, dans le groupe 3 sont inclus les patients avec une atteinte de la graisse thyroïdienne et le groupe 4 ceux présentant une atteinte des structures cervicales. L'augmentation de l'activité ablative requise est un constat qui devient pertinent lors du dépassement de la graisse péri-thyroïdienne (tableau I).

\begin{tabular}{|c|c|c|c|c|}
\hline Degré d'invasion & $\begin{array}{c}\text { Groupe 1 } \\
\text { Pas d'invasion }\end{array}$ & $\begin{array}{c}\text { Groupe 2 } \\
\text { Effraction capsulaire }\end{array}$ & $\begin{array}{c}\text { Groupe 3 } \\
\text { Atteinte de la graisse } \\
\text { pér-thyroidienne }\end{array}$ & $\begin{array}{c}\text { Groupe 4 } \\
\text { Invasion des structures } \\
\text { Cervicales }\end{array}$ \\
\hline $\begin{array}{l}\text { Activité ablative } \\
\text { moyenne ( GBq) }\end{array}$ & 5.14 & 5.79 & 6.02 & 8.32 \\
\hline
\end{tabular}

Tableau I : Valeur de l'activité ablative moyenne en fonction de l'importance de l'extension locorégionale du cancer thyroïdien à l'examen anatomopathologique

En comparant les 4 groupes de patients, on trouve que la différence de l'activité ablative moyenne est non significative entre les groupes 1,2 et 3 . Cette différence n'a été retrouvée significative qu'entre les groupes 1 et $4: p<10$ 3. Autrement, le dépassement de la graisse péri-thyroïdienne entraîne une augmentation sensible de l'activité requise pour l'ablation du reliquat. Le délai de disparition du reliquat thyroïdien augmente sensiblement avec l'importance de l'extension loco-regionale (figure 3). La différence entre les groupes 1 et 4 est de l'ordre de 8,67 mois, cette différence est statistiquement significative $(p=0,004)$.

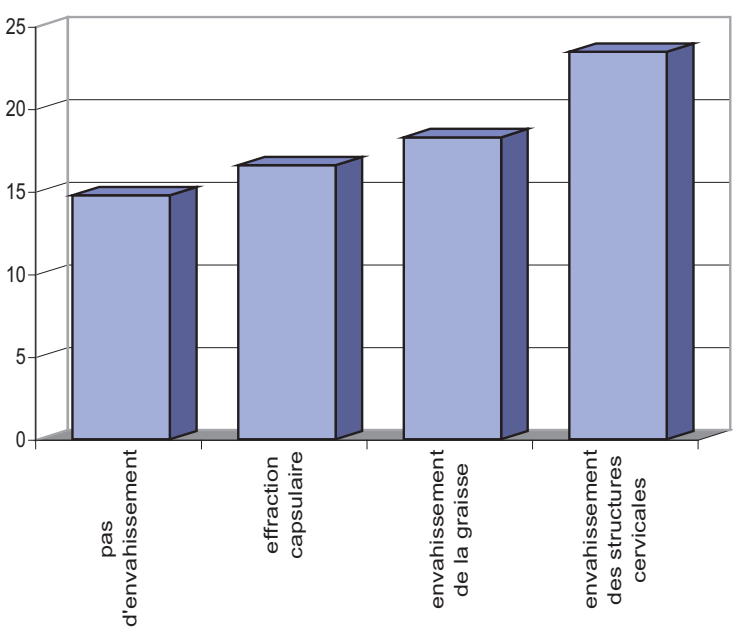

Fig. 3 : Délai de disparition du reliquat thyroïdien en fonction de l'importance de l'extension loco-regionale en mois.

- Métastases à distance : L'activité ablative moyenne est de $5,54 \mathrm{GBq}$ en l'absence de localisations métastatiques prouvées. Elle atteint les $7,03 \mathrm{GBq}$ en cas de métastases. La différence entre les deux groupes n'est pas significative. Aucune différence statistiquement significative - du délai de disparition du reliquat - n'est retrouvée entre les deux groupes.

\section{- Peut-on adopter la classification TNM comme élé- ment prédictif d'efficacité ablative ?}

La recherche de corrélation entre les différents éléments de la classification TNM et les paramètres de l'efficacité ablative trouve :

Taille tumorale : Comme nous l'avons indiqué plus haut, la valeur de l'activité ablative moyenne augmente avec la progression de la taille tumorale. Une différence significative des moyennes est notée entre les stades T3 et T4a $(p=0,041)$. Cette transition de classe s'accompagne d'une augmentation considérable de l'activité ablative. En effet, cette activité moyenne passe de $5,66 \mathrm{GBq}$ pour la classe T3, à une activité moyenne de 7,91 GBq pour la classe $\mathrm{T} 4 \mathrm{a}$ (figure 4). Un autre constat est à relever concernant le stade $\mathrm{T} 4 \mathrm{~b}$ où nous assistons à une diminution de l'activité ablative moyenne sans que la différence entre les stades $\mathrm{T} 4 \mathrm{a}$ et $\mathrm{T} 4 \mathrm{~b}$ ne soit statistiquement significative.

Atteinte ganglionnaire : L'activité ablative moyenne nécessaire pour la destruction du reliquat diffère peu entre les stades $\mathrm{N} 0$ et N1a (atteinte cervicale régionale unilatérale). Au delà du stade $\mathrm{N} 1 \mathrm{a}$, cette activité ablative moyenne augmente avec l'extension ganglionnaire. En effet, une différence d'activité moyenne de 2,4GBq est notée entre le stade (N1a) et le stade (N1b) ; cette différence est statistiquement significative $\mathrm{p}=0,009$ (figure 4). Atteinte métastatique : L'atteinte métastatique $M$ concerne les métastases viscérales à distance. Nous 
notons une différence de l'activité ablative moyenne entre les stades $\mathrm{M} 0$ et $\mathrm{M} 1$ de 1,49 GBq. Cette différence n'est pas statistiquement significative (figure 4).

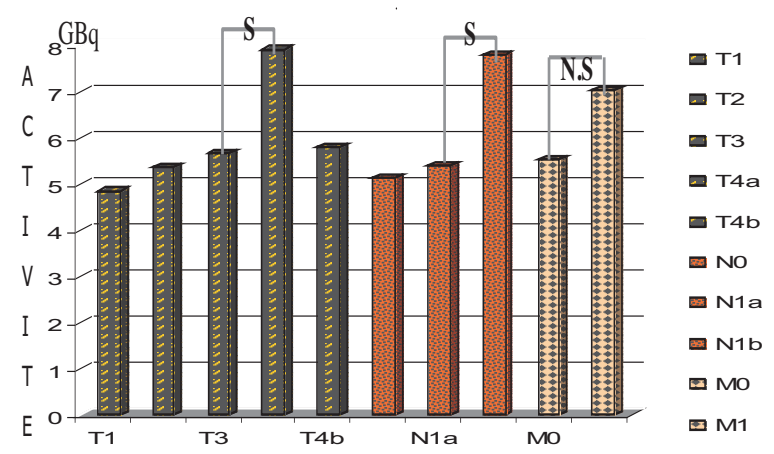

Fig. 4 : Activité ablative moyenne en fonction des différentes classes de la classification TNM

\section{- Peut-on adopter la stadification TNM comme élé- ment prédictif d'efficacité ablative ?}

La stadification TNM est une stadification pronostique qui fait appel, en plus des éléments de la classification TNM, au facteur âge (inférieur ou supérieur à 45 ans).

Nos patients étaient donc répartis en fonction du stade TNM, ainsi 169 patients appartenaient au stade I, 20 patients au stade II, 48 au stade III et 38 au stade IV (IVA, IVB et IVC confondus).

La recherche d'une corrélation entre la stadification TNM et l'efficacité ablative s'est avérée infructueuse. En effet et paradoxalement aux attentes, la valeur de l'activité ablative ne dépend pas de cette stadification TNM puisque les valeurs des doses moyennes diffèrent peu entre les stades (figure 5).

Le facteur âge utilisé pour la stadification pronostique ne semble pas modifier l'activité ablative requise, en effet la comparaison des doses ablatives moyennes pour chaque groupe d'âge utilisé pour la stadification montre des valeurs comparables : 5,4 GBq pour les sujets âgés de moins de 45 ans et $5,22 \mathrm{GBq}$ pour ceux âgés de plus de 45 ans.

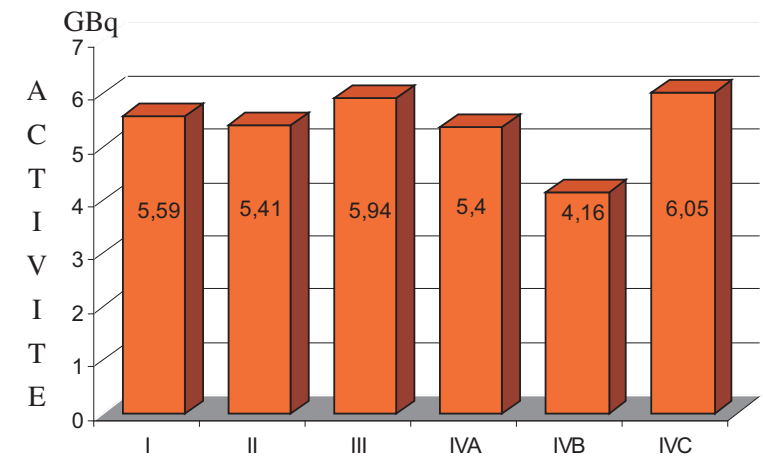

Fig. 5 : Activité ablative moyenne en fonction du stade pronostique TNM

\section{DISCUSSION}

Le traitement des CDT est basé, après la chirurgie, sur l'irathérapie. Le traitement postopératoire par l'iode 131 a pour objectifs :

- De détruire les reliquats thyroïdiens normaux et faciliter ainsi la surveillance ultérieure par le dosage de la thyroglobuline $(13,14,15)$;

- D'effectuer trois à sept jours plus tard un examen scintigraphique du corps entier qui vérifie l'absence de foyers de fixation en dehors de l'aire thyroïdienne. La sensibilité de cet examen est excellente lorsque la fixation au niveau des reliquats thyroïdiens est très faible voire absente (inférieur à $2 \%)(16,17,18)$;

- De détruire d'éventuels résidus tumoraux et diminuer ainsi le taux de rechute $(18,19,20)$.

Les deux premiers objectifs font de l'ablation isotopique une nécessité. L'efficacité de l'iode dans l'ablation des reliquats thyroïdiens dépend de plusieurs facteurs ; certains sont liés au patient (facteurs épidémiologiques, chirurgicaux, anatomopathologiques, biologiques,...) ; d'autres sont plutôt en rapport avec le traitement à l'iode radioactif (activité délivrée, délai entre les cures, ...).

Les facteurs anatomopathologiques sont à placer au premier plan puisque la sensibilité ou la résistance à l'iode radioactif dépend entre autres des cellules irradiées. Nature histologique : Selon Doudouh (21) la nature histologique ne semble pas jouer un rôle décisif dans l'efficacité du traitement. II retrouve à peu de différence près la même efficacité de l'ablation isotopique des CDT qu'ils soient de type papillaire ou vésiculaire. Ces mêmes constatations ont été retrouvées par Couette (22). Dans notre travail, nous n'avons pas trouvé de corrélation statistiquement significative entre la nature histologique du cancer et l'efficacité du traitement.

Nombre de foyer à l'examen histologique : Selon Boughattas (23), l'existence de lésions histologiques de type thyroïdite ou multinodularité associées au carcinome thyroïdien, pourrait constituer un facteur de résistance à l'iode 131. Dans son étude rétrospective portant sur 87 patients, des lésions histologiques de thyroïdite ou de multinodularité ont été notées chez 11 patients. Dans ce groupe, l'ablation a été obtenue après une première cure de $3,7 \mathrm{GBq}$ dans seulement $36 \%$ des cas, vs $61 \%$ en l'absence de ces lésions, sans que cette différence soit significative $(p=0,1)$.

Dans notre étude, l'efficacité ablative du traitement ne semble pas être liée au type de lésion retrouvée à l'examen anatomopathologique. En effet, nous avons une disparition des reliquats lorsqu'il s'agit de lésion unifocale ou plurifocale dans la plupart des cas avec des doses de $3,7 \mathrm{GBq}(63 \%$ vs $58 \%)$. Cette différence n'est pas significative.

Taille de la tumeur : Dans la littérature (24), la taille tumorale est considérée comme un facteur pronostique de la survie et du risque de récidive tumorale. Par 
contre, nous n'avons pas, à notre connaissance, d'études concernant l'implication de ce facteur dans l'efficacité de l'ablation isotopique. Notre étude montre que la taille de la tumeur peut être considérée comme un facteur prédictif de l'efficacité de l'ablation isotopique. En effet, lorsque la taille tumorale est inférieure à $5 \mathrm{~cm}$, la dose ablative ne dépasse pas les $100 \mathrm{mCi}$ dans presque $2 / 3$ des cas. L'inverse se produit lorsque la taille tumorale dépasse les $5 \mathrm{~cm}$. Cette différence est significative $(p=0,01)$

Envahissement locorégional : Le rôle présumé de l'extension locorégionale a été trouvé dans notre étude mais n'a pas été abordé dans la littérature à notre connaissance. La graisse périthyroïdienne est la frontière à ne pas dépasser pour avoir une bonne efficacité ablative.

Envahissement ganglionnaire : Dans l'étude réalisée par Doudouh (21) l'absence d'envahissement ganglionnaire peut être considérée comme un facteur prédictif d'efficacité de I'IRAthérapie avec une différence significative à 95\% de sécurité. En effet, sur les 94 malades sans localisations extra cervicales, une cartographie blanche est obtenue dès la première administration d'iode radioactif chez $67 \%$ des patients ayant un envahissement ganglionnaire contre $88 \%$ des patients ne présentant pas d'envahissement ganglionnaire. Cette constatation est confirmée également par Couette (22). Par contre, dans notre étude, nous n'avons pas trouvé de corrélation statistiquement significative entre la dose ablative utilisée et l'envahissement ganglionnaire (présent ou pas). Cependant, l'étendue de l'envahissement ganglionnaire (atteinte pluri-compartementale ou unicompartimentale) semble être un facteur prédictif d'efficacité ablative $(p=0,028)$.

Métastases à distance : Contrairement aux attentes, ce paramètre ne modifie pas l'efficacité ablative dans notre étude. Nous n'avons pas retrouvé dans la littérature d'études concernant l'influence de ce paramètre sur l'efficacité ablative.

Classification - stadification TNM : La classification anatomopathologique (pTNM) prend son origine à partir des éléments cités plus haut (26). C'est à partir de cette classification qu'est définie la stadification qui permet d'organiser ces facteurs en classes pronostiques dont les risques de rechute du CDT et de la mortalité liée au cancer sont différents. Cette stadification ainsi définie fait appel également à l'âge comme élément pronostique principal. Ce dernier est excellent quand le sujet est jeune, même en cas d'adénopathies métastatiques (stade I : environ $95 \%$ de survie à 10 ans) et est moins bon lorsqu'il est plus âgé. Néanmoins des survies prolongées peuvent être obtenues même à des stades avancés.

Malgré le rôle supposé de la classification TNM pour prédire l'activité ablative du reliquat post opératoire, la stadification proposée n'est d'aucun apport dans ce cadre comme montré dans notre étude. Ainsi, et contrairement au pronostic, la prédiction de l'efficacité ablative de l' I 131sur le reliquat thyroïdien ne peut être établie sur les mêmes critères.

L'absence de corrélation entre l'âge des patients et l'efficacité de l'activité ablative est sans doute le principal élément expliquant l'échec de l'utilisation de cette stadification.

D'autres facteurs tel que les facteurs épidémiologiques, les facteurs biologiques ( $\mathrm{Tg}$ ) ainsi que le bilan scintigraphique initial pré-irathérapie (résultat du BCE diagnostique) devraient être pris en considération.

\section{CONCLUSION}

La prédiction de l'efficacité ablative et donc de l'activité requise pour détruire le reliquat thyroïdien dépend de la taille tumorale et de l'extension lymphatique, locorégionale et à distance (métastases).

Une taille tumorale inférieure à $5 \mathrm{~cm}$, l'atteinte lymphatique au plus mono-compartimentale et l'absence d'invasion de la graisse péri-thyroïdienne prédisent une meilleure efficacité ablative de l'iode 131.

Cette efficacité peut être traduite dans la classification pTNM, ce qui correspond à $\mathrm{T}<\mathrm{T} 3$ et $\mathrm{N}<\mathrm{N} 1 \mathrm{~b}$ mais pas du stade pronostique initial de la tumeur. II serait donc judicieux de proposer une nouvelle stadification d'efficacité ablative basée sur les critères pTNM cités plus haut et englobant en plus, à la place de l'âge, le résultat du BCE à l'iode 131 et de la $\mathrm{Tg}$ initiales. D'autres études prospectives, randomisées seraient nécessaires pour déterminer le meilleur agencement de ces critères. 


\section{REFERENCES}

1- Pacini F, Schlumberger M, et al. European consensus for the management of patients with differentiated thyroid cancer of the follicular epithelium. Eur $\mathrm{J}$ Endocrinol 2006; 154: 787-803.

2- Sweeney D.C, Johnston G.S. Radioiodine therapy for thyroid cancer. Endocrinol. Metab. Clin. NA. 1995; 24: 803-839

3- Maheshwari Y K, hill C S, Haynie T P et Al. 131 I therapy in the differentiated thyroid carcinoma. Cancer 1981; 47: 667-671

4- Causeret S, Lifante J. C, Borson-Chazot F et al. Cancer différencié de la thyroïde chez l'enfant et l'adolescent: stratégie thérapeutique adaptée a la présentation clinique. Annales de chirurgie. 2004; 129 (6-7): 359-364

5- Beirwaltes W, Rabbani R, Dmuchowski and Al. An analysis of "Ablation of thyroid remnants" with iode 131 in 511 patients from 1947-1984: experience at university of Michigan. J. Nucl. Med. 1984; 25: 1287-1293

6 - Schlumberger M. Cancer papillaire et folliculaire de la thyroïde. Encyclopédie Orphanet. Annales d'Endorinologie 2007; 68: 120-128

7- Reiners C, Dietlein M, Luster M. Radio-iodine therapy in differentiated thyroid cancer: indications and procedures. Best Pract Res Clin Endocrinol Metab. 2008; 22(6): 989-1007.

8- Pacini F, Sclumberger M, Harmer $\mathrm{C}$ et al. Post-surgical use of radioiodine (131I) in patients with papillary and follicular thyroid cancer and the issue of remnant ablation: a consensus report. Eur J Endocrinol 2005; 153(5): 651-659.

9- Menegaux F, Leenhardt L, Hoang C, Chigot J-P. Chirurgie du cancer thyrö̈dien.

Médecine thérapeutique : actualité en chirurgie. 2001; 7(3): 192-200.

10- Rassael H, Thompson L, Heffess C. A rationale for conservative management of microscopic papillary carcinoma of the thyroid gland: a clinicopathological correlation of 90 cases. Eur Arch Otorhinolaryngol. 1998; 255: 462-467. 11- Furlan JC. Bedard Y. Rosen IB. Biologic basis for the traitement of microscopic, occult well differentiated thyroid cancer. Surgery. 2001; 130 (6): 1050-4 12- Schlumberger M, Baudin E, Travagli JP. Papillary and follicular cancers of the thyroid. Presse Med. 1998; 27(29): 1479-81.

13- Cooper DS, Doherty GM, Haugen BR, et al. Management guidelines for patients with thyroid nodules and differentiated thyroid cancer. Thyroid 2006; 16 : $109-42$
14- Schlumberger M. Can iodine-131 whole- body scan be replaced by thyroglobuline measurement in the post-surgical follow-up of differentiated thyroid carcinoma? J Nucl Med. 1992; 33(1): 172-3

15- Schluberger M., Tubiana M, De Vathaire F, and Al. Long-term results of treatement of 283 patients with lung and bone metastases from differentiated thyroid carcinoma. J Clin Endocrinol Metab. 1986; 63(4): 960-7.

16- Sweeney D, Johnston G. Radioiodine therapy for thyroide cancer. Endorinal Metb. Clinics North AM. 1995; 24(4): 803-839.

17- Galloway R, Smalridge R. Imaging in thyroid cancer. Endocrinol. Metab. Clinic North AM 1996; 25: 93-113

18- Boulahdour $\mathrm{H}$, Mellière $\mathrm{D}$, Brugières $\mathrm{P}$ et Al. Microcancers différenciés de la thyroïde : faut-il utiliser le radioiode ? Médecine Thérapeutique Endocrinologie \& Reproduction. 2002; 4(1): 63-65

19- Samaan NA, Schultz PN, Hickey RC and Al. The results of various modalities of treatment of well differentiated thyroid carcinoma: A retrospective review of 1599 patients. J.Clin. Endocrinol. Metab. 1992; 75(2): 714-720.

20- Chow SM, Law SC, Chan JK, Au SK and Al. Papillary microcarcinoma of the thyroid-prognostic significance of lymph node metastasisand multifocality. Cancer. 2003; 98(1): 31-40

21 Doudouh A, Biy A, Zekri A et Al. Traitement par l'iode 131 des résidus cervicaux dans les cancers différenciés de la thyroïde. Médecine du Maghreb 2006; 142: $23-27$

22- Couette J.E, Van H.L., Harang H. et al. Evaluation de l'efficacité de la thérapie par iode131 sur les résidus cervicaux dans les cancers thyroidiens différenciés. Médecine nucléaire et cancérologie 1995; 19(2-3): 116-119

23- Boughattas S. Le fractionnement des activités ablatives diode 131 dans les carcinomes différenciés de la thyroïde. Revue de l'ACOMEN 1998; 4(3): 198201

24- Leclere J, Klein M. Les facteurs pronostiques dans les cancers différentiés de la thyroide (médullaire exclus) Ann Endocrinol (Paris) 1997; 58: 183-187 25- Loh KC, Greenspan FS, Gee L, et al. Pathological tumor-node-metastasis (pTNM) staging for papillary and follicular thyroid carcinomas: a retrospective analysis of 700 patients. J Clin Endocrinol Metab. 1997; 82(11): 3553-3562. 